ARTICLE

\title{
Diffusion engineering of ions and charge carriers for stable efficient perovskite solar cells
}

Enbing $\mathrm{Bi}^{1,2, \star}$, Han Chen ${ }^{1, \star}$, Fengxian $\mathrm{Xie}^{2, \star}$, Yongzhen $\mathrm{Wu}^{2}$, Wei Chen ${ }^{2}$, Yanjie Su${ }^{3}$, Ashraful Islam ${ }^{2}$, Michael Grätzel ${ }^{4}$, Xudong Yang ${ }^{1} \&$ Liyuan $\operatorname{Han}^{1,2}$

Long-term stability is crucial for the future application of perovskite solar cells, a promising low-cost photovoltaic technology that has rapidly advanced in the recent years. Here, we designed a nanostructured carbon layer to suppress the diffusion of ions/molecules within perovskite solar cells, an important degradation process in the device. Furthermore, this nanocarbon layer benefited the diffusion of electron charge carriers to enable a high-energy conversion efficiency. Finally, the efficiency on a perovskite solar cell with an aperture area of $1.02 \mathrm{~cm}^{2}$, after a thermal aging test at $85^{\circ} \mathrm{C}$ for over $500 \mathrm{~h}$, or light soaking for $1,000 \mathrm{~h}$, was stable of over $15 \%$ during the entire test. The present diffusion engineering of ions/molecules and photo generated charges paves a way to realizing long-term stable and highly efficient perovskite solar cells.

\footnotetext{
${ }^{1}$ State Key Laboratory of Metal Matrix Composites, School of Materials Science and Engineering, Shanghai Jiao Tong University, Shanghai 200240, China. ${ }^{2}$ Photovoltaic Materials Unit, National Institute for Materials Science, Tsukuba, Ibaraki 305-0047, Japan. ${ }^{3}$ Key Laboratory for Thin Film and Microfabrication of the Ministry of Education, Department of Micro/Nano Electronics, School of Electronics, Information and Electrical Engineering, Shanghai Jiao Tong University, Shanghai 200240, China. ${ }^{4}$ Laboratory of Photonics and Interfaces (LPI), Station 6, Institute of Chemical Science and Engineering, Faculty of Basic Science, Ecole Polytechnique Federale de Lausanne, CH-1015 Lausanne, Switzerland. * These authors contributed equally to this work. Correspondence and requests for materials should be addressed to M.G. (email: michael.graetzel@epfl.ch) or to X.Y. (email: yang.xudong@sjtu.edu.cn)

or to L.H. (email: han.liyuan@nims.go.jp).
} 
$\mathrm{H}$ ybrid organic-inorganic perovskite materials are the subject of intense current investigations owing to their excellent photovoltaic properties, for example, intense visible (vis) light absorption, tuneable band gap and long charge carrier diffusion length ${ }^{1-7}$. The perovskite solar cells (PSCs) have been considered as the most competitive next generation photovoltaic technology that exhibits the advantages of low-cost processing, abundantly available materials and unprecedented rise in solar to electric power conversion efficiency (PCE) (refs 8-14). The state-of-the-art PSCs reached a PCE of $22 \%$ (ref. 14) and demonstrated the feasibility of achieving PCEs of over $25 \%$ for tandem cells ${ }^{15}$. However, the long-term stability of PSCs is notoriously poor, which has been considered as one of the major challenges for future large-scale application ${ }^{16-19}$.

The long-term stability of PSCs has been restricted owing to the low-energy barrier for ions/molecules migration within perovskite light absorbers whose crystal lattice can be easily changed or collapsed under thermal activation, light soaking, moisture invation ${ }^{9,20}$, photo-induced reaction ${ }^{21}$, or phase separation $^{22,23}$ etc., Among the tremendous efforts to suppress the degradation, some approaches have successfully improved the device stability, including the introduction of carbon counter electrode $^{9}$, composition engineering with mixed anions ${ }^{11}$ or cations ${ }^{15}$, heavily doped metal oxide as robust charge transporting materials ${ }^{12}$, surface passivation ${ }^{24}$, hydrophobic materials ${ }^{25}$ and crystal growth with reduced defects ${ }^{26}$ etc., However, it is still a big challenge to achieve highly efficient PSCs exhibiting long-term stable performance.

Here, we provide a strategy for high efficiency and long-term stability via diffusion engineering to hinder unfavourable ions/molecules diffusion and accelerate photo generated charges diffusion within PSCs. We deposited a nanostructured carbon layer acting as an ions/molecules blocking and electron extraction layer (EEL), containing $\mathrm{N}$-doped graphene, the fullerene derivative phenyl-C61-butyric acid methyl ester (PCBM) and carbon quantum dots (CQDs), between the perovskite light absorber layer and the electrode layer. In comparison with conventional strategy of increasing the thickness of blocking layers, the present strategy enabled a higher capability of almost 3 times of that of conventional EELs in blocking ions/molecules diffusion at the same thickness. The major contribution was attributed to the graphene derivatives in the nanocarbon EEL. The layer-to-layer diffusion of iodide from iodide-rich perovskite layer to the electrode layer was successfully hindered for both of the $\mathrm{CH}_{3} \mathrm{NH}_{3}$ (methylammonium or MA) or $\mathrm{HC}\left(\mathrm{NH}_{2}\right)_{2}$ (formamidinium or FA) based perovskite materials, where such diffusion has been reported as a serious device degradation or defects generation process causing poor device stability ${ }^{16,27,28}$. This undesirable diffusion is related to the relatively low activation energy for iodide migration and the high iodide concentration gradient of about $10^{26} \mathrm{~cm}^{-4}$ within a tiny distance of tens of nanometers from the perovskite surface to the electrode, which can be further accelerated at a higher temperature ${ }^{16,23,27-30}$. The diffusion of Ag atoms from electrode to perovskite layer, another factor for the device degradation ${ }^{31}$, was also retarded by the present nanocarbon layer. In addition, the designed nanocarbon layer also exhibited a high electric conductivity and good ohmic contact with the metal electrode. As a result, we were able to obtain centimetre-squared device with a certified efficiency of $15.6 \%$ recorded in the solar cell efficiency tables $^{32}$. Our work also presented the PSCs had good thermal stability and light stability, which exhibited a stable efficiency of over $15 \%$ during thermal aging test at $85^{\circ} \mathrm{C}$ for $500 \mathrm{~h}$ or light soaking under air mass 1.5 global (AM 1.5G) illumination for $1,000 \mathrm{~h}$. This strategy provides a promising way to the future practical application of highly stable and efficient large-area PSCs.

\section{Results}

Device structure. We prepared PSCs with a planar heterojunction structure of layers superimposed in the sequence FTO/NiMgLiO (20 nm)/MAPbI 3 (350 nm)/G-PCBM (50-200 nm)/ CQDs $(10 \mathrm{~nm}) / \mathrm{Ag}(100 \mathrm{~nm})$ (Fig. 1a). G-PCBM stands for PCBM doped with $2 \mathrm{wt} \% \mathrm{~N}$-doped graphene to block the layer-to-layer diffusion of iodide or water molecules (Fig. 1b). The work function for CQDs was derived from UPS measurements (Supplementary Fig. 1) showing the conduction band of CQDs to be positioned at $-4.1 \mathrm{eV}$ (Fig. 1c).

Iodide diffusion. To scrutinize the influence of the nanocarbon layer on the layer-to-layer diffusion of iodide, we compared the $\mathrm{UV}$-vis absorption spectra of $\mathrm{MAPbI}_{3}$ perovskite films coated with different nanocarbon layers (Supplementary Fig. 2) and used the light absorbance at $600 \mathrm{~nm}$ to assess the fraction of the perovskite materials that remained intact. All samples were put on a hotplate at $100^{\circ} \mathrm{C}$ in $\mathrm{N}_{2}$ for thermal aging test to accelerate the diffusion process. The perovskite film exposed to $\mathrm{N}_{2}$ was almost decomposed within $15 \mathrm{~h}$ under thermal heating. The reason for the thermal decomposition of perovskite film is that the activation barrier of migration of iodide to its vacancy is relatively small ${ }^{29,30}$. The perovskite film can be thermally decomposed to $\mathrm{PbI}_{2}$ and $\mathrm{MA}$, and $\mathrm{HI}$, where the $\mathrm{MA}$ and $\mathrm{HI}$ can escape from the perovskite surface and left iodide vacancies. At a higher temperature, the iodide has a higher energy to enable a faster diffusion from the inside of the perovskite film to the surface vacancies. With the increase of vacancy defects at the surface, the movement of iodide within the perovskite film and the consequent degradation will be accelerated. In contrast,
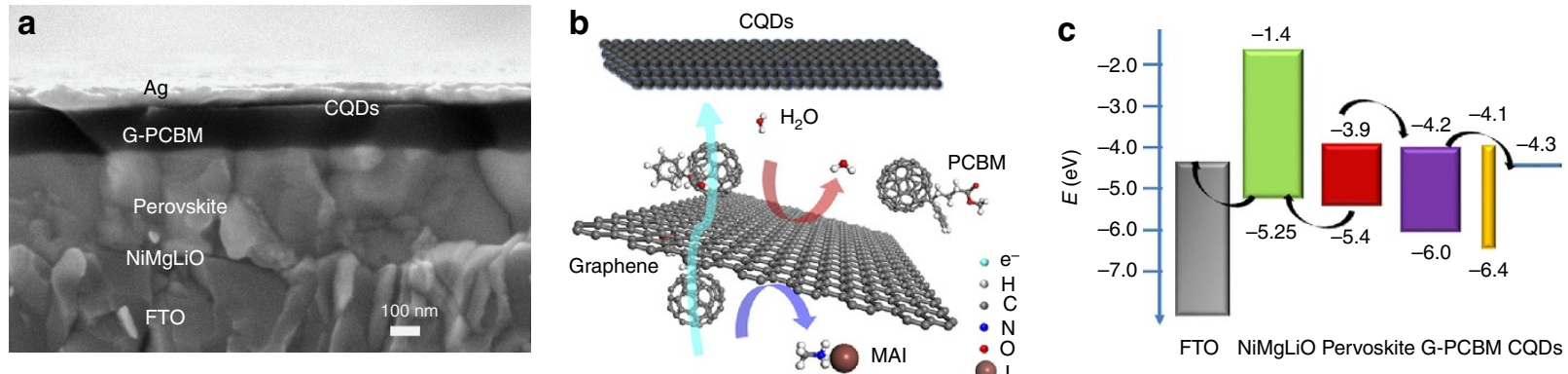

FTO NiMgLiO Pervoskite G-PCBM CQDs Ag

Figure 1 | Planar heterojunction PSCs using a nanocarbon layer to suppress layer-to-layer ions/molecules diffusion and facilitate electron charge carrier diffusion. (a) SEM cross-section image of a NiMgLiO/Perovskite/N-doped graphene fullerene derivative phenyl-C61-butyric acid methyl ester (G$\mathrm{PCBM}$ )/CQDs/Ag device, in which G-PCBM is graphene doped PCBM. (b) Schematic drawing of the diffusion processes within the nanocarbon-based EEL. (c) Energy band structure of the planar heterojunction cell. 
for the $\mathrm{MAPbI}_{3}$ film covered with $150 \mathrm{~nm}$ G-PCBM, the light absorbance at $600 \mathrm{~nm}$ lost only $8 \%$ after 180 ageing hours, which was almost 3 times longer than that of the samples covered with PCBM film at the same thickness. The thermal stability of $\left(\mathrm{FAPbI}_{3}\right)_{0.85}\left(\mathrm{MAPbBr}_{3}\right)_{0.15}$ perovskite film was also greatly enhanced when covered with the G-PCBM film (Supplementary Fig. 3). These results strongly suggest that the graphene derivatives in the present nanocarbon layer can effectively hinder the loss of iodide from the perovskite film and the consequent degradation in comparison with conventional PCBM EELs.

As the diffused iodide across the EEL could corrode the silver electrode, we further determined the spatial distribution of iodide in thermally aged PSCs. The scanning electron microscopy with energy dispersive X-ray (SEM-EDX) analysis (Fig. 2a-c) indicate that the iodide diffused across the EEL to the silver electrode in $50 \mathrm{~nm}$ PCBM based device while it was almost hindered in $150 \mathrm{~nm}$ PCBM or G-PCBM based device. In Fig. 2d, the X-ray photoelectron spectroscopy (XPS) spectra shows the signal from iodide in silver electrode of the $150 \mathrm{~nm}$ G-PCBM based device was the smallest and only one-third of that of the $150 \mathrm{~nm}$ PCBM based device, indicating the graphene derivatives blocking the iodide diffusion effectively. In addition, the silver electrode was smooth than the rough one in the $50 \mathrm{~nm}$ PCBM based devices that was caused by the iodide corrosion (Fig. 2a,e). We note that the iodide, with ions diameter of $0.412 \mathrm{~nm}$, cannot penetrate the two-dimensional crystal lattice of graphene (lattice parameter $a=0.246 \mathrm{~nm}$ ). So in a G-PCBM layer, the diffused iodide has to move round the graphene derivatives (the typical size is of about $100 \mathrm{~nm}$ ). The total diffusion path of iodide or other molecules in G-PCBM EEL with a typical thickness of $150 \mathrm{~nm}$ would be extended about 10 times than that of $50 \mathrm{~nm}$ PCBM EEL. According to Fick's law, a long diffusion length can reduce the concentration gradient and consequently the diffusion flux, which can hinder the diffusion of iodide out from the perovskite film and consequent decomposition. Another possible reason for the decomposition of perovskite film is the diffusion of $\mathrm{Ag}$ into perovskite film ${ }^{31}$. The $\mathrm{Ag}$ diffusion would be enhanced if the nanocarbon layer cannot fully cover the surface of perovskite film that may make perovskite/Ag contact. We used a solvent engineering method to deposit smooth perovskite film with roughness lower than $20 \mathrm{~nm}$ so that the nanocarbon layer with thickness of $50 \mathrm{~nm}$ or larger can fully cover the perovskite films ${ }^{33}$. As shown in Supplementary Fig. 4, the perovskite film covered with $\mathrm{Ag}$ electrode, in the perovskite/PCBM $(150 \mathrm{~nm}) / \mathrm{CQDs} / \mathrm{Ag}$ cell, lost the typical dark-brown colour and turned to be yellow while the perovskite film without $\mathrm{Ag}$ showed less change, which indicate the perovskite degraded faster than that without $\mathrm{Ag}$ electrode. In comparison, the perovskite film covered with G-PCBM $(150 \mathrm{~nm}) / \mathrm{CQDs} / \mathrm{Ag}$ film was much stable with negligible change after the same ageing test. This result strongly suggests that the present nanocarbon layer is desired as a more effective barrier to prevent direct perovskite/Ag contact and suppress Ag or iodide diffusion from layer-to-layer.

Device stability. The device stability was then studied to evaluate the effects of different nanocarbon layers. As shown in Fig. 3a, the best of the optimized devices using CQD/G-PCBM $(150 \mathrm{~nm})$ exhibited a small hysteresis in current-voltage $(J-V)$ characteristics with a PCE of $17.0 \%$ at forward scan and $17.4 \%$ at reverse scan under simulated AM 1.5G solar light with an aperture area of $1.02 \mathrm{~cm}^{2}$ (Supplementary Fig. 5). This device performed stably during a short-term test of $200 \mathrm{~s}$ (Supplementary Fig. 6). We measured the incident photon-to-current conversion efficiency (IPCE) spectrum (Fig. 3b) and the integrated $J_{\mathrm{SC}}$ matched well with the value in $J-V$ characteristics. For the long-term stability test of sealed cells, the PCE of a CQDs/G-PCBM $(150 \mathrm{~nm})$ based device was stable of over $15 \%$ when the device had been kept under dark at room temperature for $5,000 \mathrm{~h}$ or under AM 1.5G simulated solar light for $1,000 \mathrm{~h}$ (Fig. 3c). Particularly, during the thermal ageing test at $85^{\circ} \mathrm{C}$ for $500 \mathrm{~h}$, the PCE was also stable of over $15 \%$ with remaining $98 \%$ of the initial value (Fig. $3 \mathrm{~d}$ and Supplementary Fig. 7). In contrast, the device with the same thickness PCBM layer showed obvious degradation with a loss of $20 \%$ of the initial PCE, where a worse light absorbance of a decomposed perovskite film reduced the photocurrent density and a worse Ag electrode lowered the fill factor. We highlighted the stable efficient PSCs with an efficiency of over 15\% during long-term thermal ageing and light soaking test, which indicates our designed nanocarbon layer plays the key role in the device stability.

The unsealed devices with CQDs/G-PCBM layer also enabled a better stability than other ones (Supplementary Fig. 8a). We further compared unsealed devices using CQDs, LiF (the work function is $3.8 \mathrm{eV}$ ) or $\mathrm{Ca}$ (the work function is $2.9 \mathrm{eV}$ ) as the interlayer between PCBM and silver. The PCE of LiF or Ca based devices dropped more than $30 \%$ or $60 \%$ due to their sensitivity to air and water. By contrast, the CQDs based devices showed less degradation of $17 \%$ than $\mathrm{LiF}$ and Ca based devices during ageing test (Supplementary Fig. 8b). Therefore, the CQDs/G-PCBM layer can suppress the diffusion of iodide escaping from the iodide-rich perovskite layer and the diffusion of molecules from air into the device, which is a key advantage for the realization of stable PSCs by suppressing the generation of defects.

The effect of layer thickness of CQDs/PCBM and CQDs/G-PCBM on the device performance were studied (Supplementary Fig. 9, Supplementary Tables 1 and 2). Although CQDs/PCBM (lower than $50 \mathrm{~nm}$ ) based devices showed high performance with PCE more than $15 \%$, the efficiencies varied over a wide range (Supplementary Fig. 10) indicating poor reproducibility in producing $1-\mathrm{cm}^{2}$-sized PSCs. The devices with CQDs/PCBM films showed better reproducibility, however, the PCEs were low because of the loss in $\mathrm{FF}$ and $J_{\mathrm{SC}}$. On the contrary, the PCEs of CQDs/G-PCBM devices were relatively insensitive to its thickness, implying a good charge transport within the CQDs/G-PCBM layer.

We then compared the electronic conductivity of PCBM and G-PCBM films by scanning probe microscope (Supplementary Fig. 11). At a bias potential of $1 \mathrm{~V}$, the average of electric current through the $150 \mathrm{~nm}$ G-PCBM EEL was $23.1 \mathrm{nA}$, which was 5.6 times as large as that of $4.1 \mathrm{nA}$ for the PCBM reference of the same thickness. These films also exhibited trap-free space charge limit current (SCLC) in the bias range of $0.1-1.0 \mathrm{~V}$ (Supplementary Fig. 11e). Using the Mott-Gurney law and Einstein relation to express the voltage dependence of the current density:

$$
J=9 \varepsilon_{r} \varepsilon_{0} D_{\mathrm{e}} V^{2} / 8 k_{\mathrm{B}} T L^{3}
$$

where $J$ is current density, $\varepsilon_{r}$ is relative dielectric constant of PCBM and $\varepsilon_{0}$ is the vacuum permittivity, $D_{\mathrm{e}}$ is the electron diffusion coefficient, $V$ is applied voltage, $k_{\mathrm{B}}$ is the Boltzmann constant, $T$ is the temperature in kelvin and $L$ is the thickness of the layer, we derived electron diffusion coefficients, of $7.80 \times 10^{-5} \mathrm{~cm}^{2} \mathrm{~s}^{-1}$ and $1.48 \times 10^{-5} \mathrm{~cm}^{2} \mathrm{~s}^{-1}$ for the G-PCBM and PCBM films respectively. This agrees well with the results obtained from SPM and follows the observed trends in device performance (Supplementary Fig. 9c). For pristine PCBM films, the phase image clearly showed the aggregates of PCBM with the size ranging from 20 to $50 \mathrm{~nm}$ (Supplementary Fig. 12). The values of $R_{\mathrm{ms}}$ of PCBM film and G-PCBM were 6 and $1 \mathrm{~nm}$, respectively, both of which were much less than the preferred 

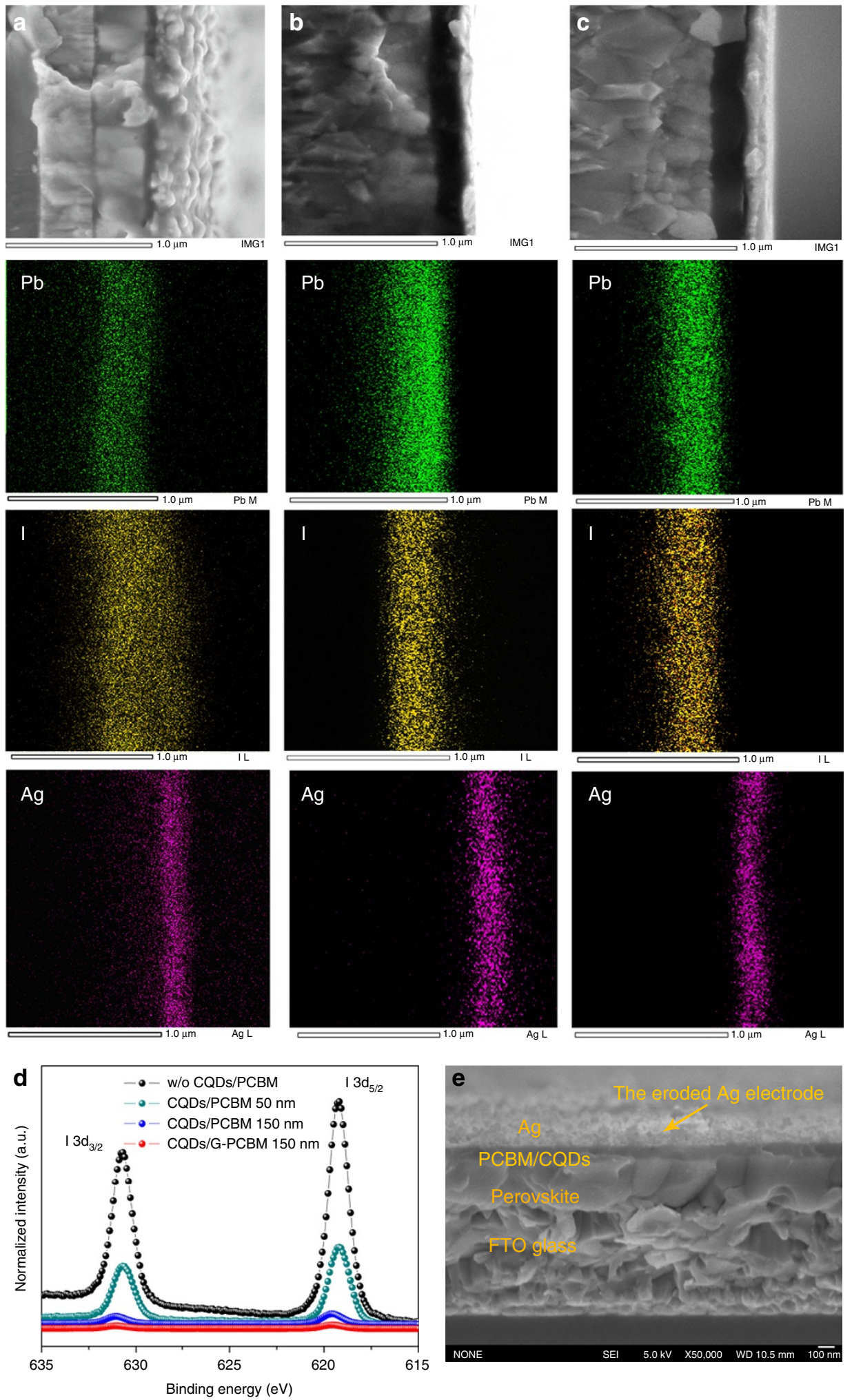

Figure 2 | Monitoring iodide distribution in PSCs. (a-c) SEM-EDX analysis of PSCs based on CQDs/fullerene derivative phenyl-C61-butyric acid methyl ester (PCBM) $(50 \mathrm{~nm}), C Q D s / P C B M(150 \mathrm{~nm})$ and CQDs/N-doped graphene fullerene G-PCBM $(150 \mathrm{~nm})$, respectively. The PSCs were preheated at temperature of $100^{\circ} \mathrm{C}$ in the dark and dry conditions for $90 \mathrm{~h}$. (d) XPS spectra of iodide at silver electrode. (e) SEM image of cross-section of PCBM $(50 \mathrm{~nm})$ based device after thermal aging in the ambient environment for 7 days.

thickness of $150 \mathrm{~nm}$. The slightly improved morphology may also contribute a little to suppress the ions migration. Thus, from the phase and surface topology of the PCBM and G-PCBM films, we infer that the G-PCBM EEL formed a compact layer which assists electron transport while impairing penetration of ions or water.

We evaluated the electron extraction dynamics via steady-state photoluminescence (PL) and time-resolved photoluminescence 
(TRPL) measurements (Fig. 4a,b). For the $\mathrm{MAPbI}_{3}$ film alone, a long lifetime of more than $100 \mathrm{~ns}$ was observed. This suffices to ensure a carrier diffusion length of hundreds of nanometers, comparable to the thickness of the light harvesting film ${ }^{4}$. The PL lifetime for the perovskite/PCBM film was $10.8 \mathrm{~ns}$, which is consisted with a previous report ${ }^{28}$. The perovskite/G-PCBM a
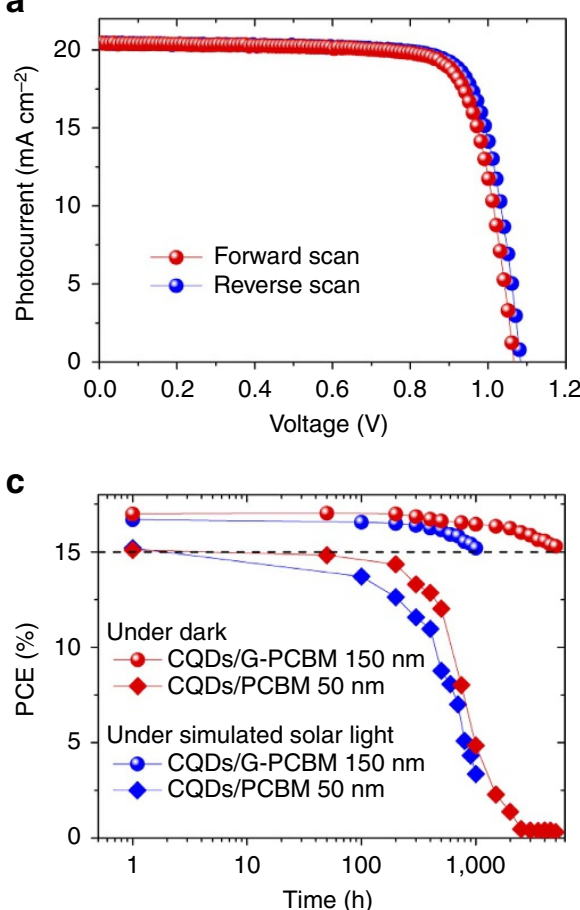

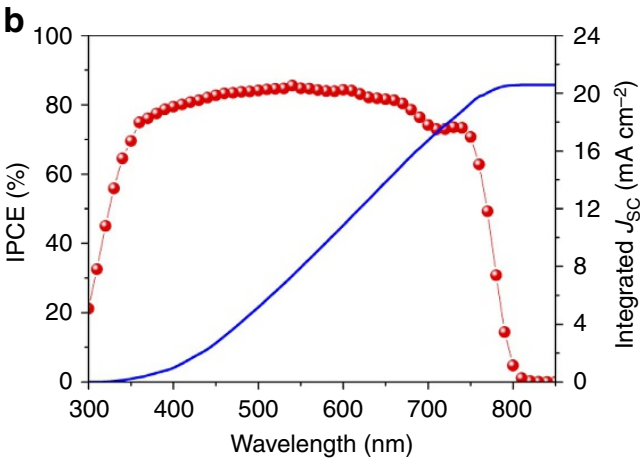

d

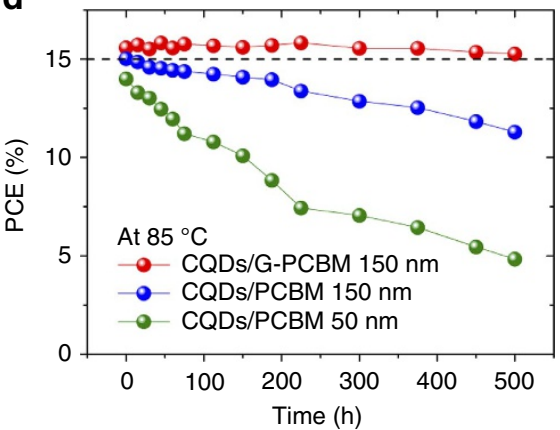

Figure 3 | Performance of PSCs. (a) The photocurrent current-voltage characteristics of one device with the structure of NiMgLiO/Perovskite/N-doped graphene fullerene derivative phenyl-C61-butyric acid methyl ester (G-PCBM) (150 nm)/CQDs (10 nm)/Ag, measured under simulated solar light (AM 1.5G, $100 \mathrm{~mW} \mathrm{~cm}^{-2}$ ). (b) Its IPCE spectrum. (c) The stability of sealed devices under dark and under air AM 1.5G simulated solar light. (d) The stability of sealed cells in thermal ageing test at $85^{\circ} \mathrm{C}$ in an atmosphere with relative humidity of about $50 \%$.
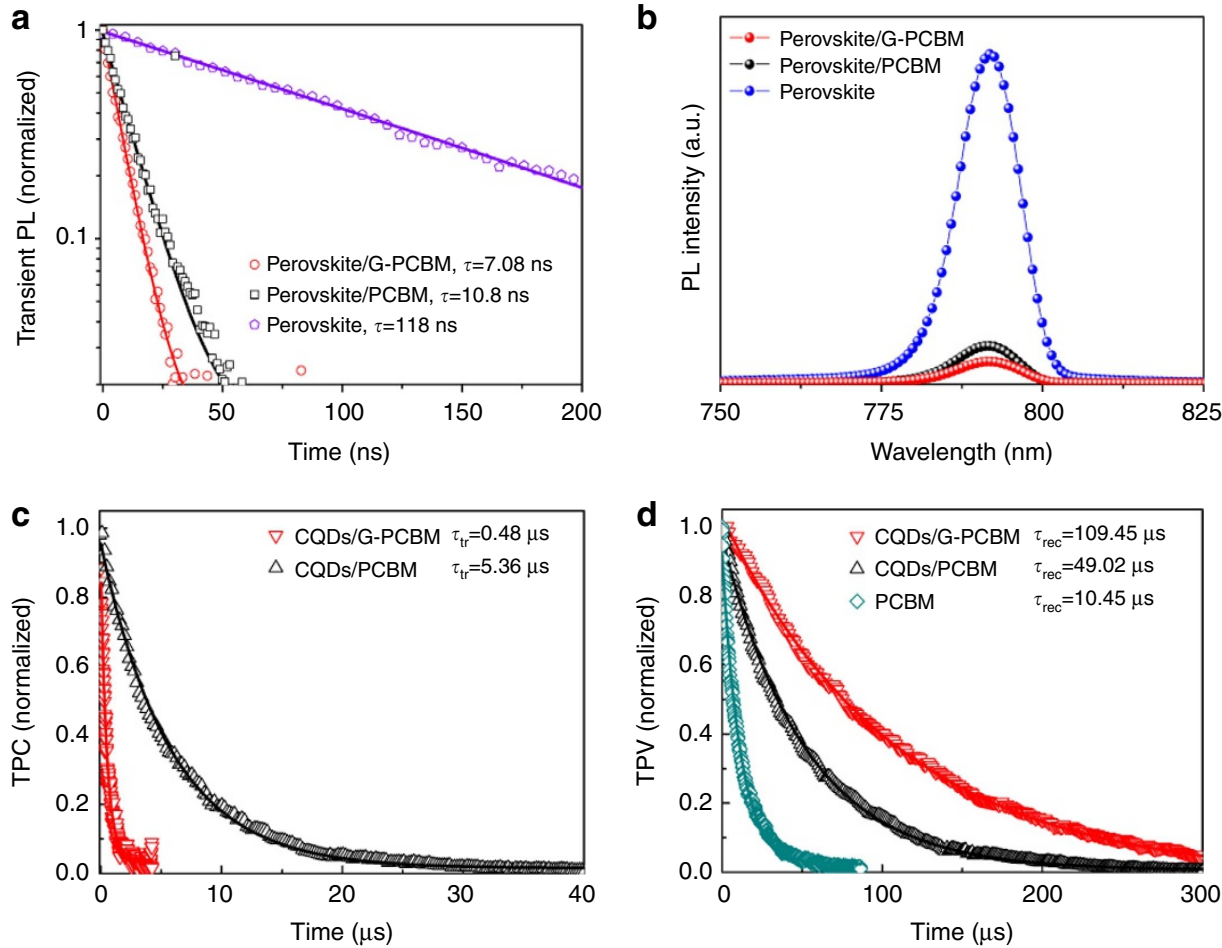

Figure 4 | Electron extraction and charge recombination properties. (a,b) TRPL decay curves and steady-state PL of MAPbl films covered by fullerene derivative phenyl-C61-butyric acid methyl ester (PCBM) and N-doped graphene G-PCBM. Solid lines are fitted results with a double exponential decay. $(\mathbf{c}, \mathbf{d})$ The transient photocurrent and photovoltage decay curves of $\mathrm{MAPbl}_{3}$ devices with CQDs/PCBM and CQDs/G-PCBM, respectively. 
showed the PL lifetime of being shortened to $7.08 \mathrm{~ns}$, indicating a faster charge extraction. Correspondingly, a strong quenching of the steady-state PL was observed for the perovskite/G-PCBM film than perovskite or perovskite/PCBM film. The electron capture is likely to be accelerated by the faster electron transport in the G-PCBM compared to the reference PCBM film ${ }^{34,35}$.

The charge transport and recombination time constants ( $\tau_{\text {tr }}$ and $\tau_{\text {rec }}$ ) were derived from the transient photocurrent and voltage measurements performed under short-circuit and opencircuit conditions, respectively (Fig. 4c,d). The CQDs/G-PCBM based devices exhibited a much more rapid photocurrent decay ( $\tau_{\text {tr }}$ of about $\left.0.48 \mu \mathrm{s}\right)$ than that of the CQDs/PCBM ( $\tau_{\text {tr }}$ of about $5.36 \mu \mathrm{s}$ ), confirming the faster electron transport in the CQDs/ G-PCBM film. This was in good agreement with our observation that the electron diffusion coefficient in G-PCBM was about 5.3 times larger than that in PCBM. The CQDs/G-PCBM $(150 \mathrm{~nm})$ device with a $V_{\mathrm{OC}}$ of $1.09 \mathrm{~V}$ exhibited slower photovoltage decay ( $\tau_{\text {rec }}$ of about $109.45 \mu \mathrm{s}$ ), indicating a better retardation of carrier recombination compared to that of a CQDs/PCBM $(150 \mathrm{~nm})$ device $\left(\tau_{\text {rec }}\right.$ of about $49.02 \mu \mathrm{s}, V_{\mathrm{OC}}$ of $\left.1.05 \mathrm{~V}\right)$. We also compared the recombination properties of a CQDs/PCBM with the PCBM reference. The photovoltage decay time in an aged device with a neat $50 \mathrm{~nm}$ PCBM film (with stable $V_{\mathrm{OC}}$ of $0.86 \mathrm{~V}$ ) was $10.45 \mu \mathrm{s}$, which is much shorter than that observed in CQDs/PCBM device. Thus, the present nanocarbon layer contributed to the suppression of the unwanted carrier recombination process. As a result of the improvement in charge transport and recombination, CQDs/G-PCBM devices exhibited superior PCEs than CQDs/ PCBM devices at the same thickness ranged from 50 to $200 \mathrm{~nm}$.

We also investigated the effect of the CQD layer thickness on the PV metrics and present data in Supplementary Table 3. The PCE was improved up to $15.8 \%$ for devices with CQDs thickness of $10 \mathrm{~nm}$, much larger than that of $8.1 \%$ for a CQD-free device. As shown in Supplementary Fig. 8d, the S-shaped $J-V$ curves of CQD-free cells in the forward bias range from 0.6 to $1.0 \mathrm{~V}$ indicated the formation of a Schottky-barrier at the PCBM/Ag junction $^{36}$. The application of CQDs buffer layer rectified the shape of $J-V$ curves and improved both the $F F$ and $J_{\mathrm{SC}}$ (Supplementary Table 4). Hence apart from retarding charge carrier recombination, the CQDs eliminated the Schottky-barrier at the junction of PCBM/Ag by forming an ohmic contact that facilitated the electron transport through the interface. We sent one of our cells to a public test centre for certification, which obtained a PCE of $15.6 \%$ (Supplementary Fig. 13). This was also recorded for certified PSCs with an aperture area of more than $1.0 \mathrm{~cm}^{2}$ (ref. 24).

\section{Discussion}

In summary, we demonstrated an approach for highly stable efficient PSCs by controlling the diffusion process of charge carriers and ions/molecules within PSCs with a nanocarbon layer consisting of graphene derivatives, fullerene derivatives and CQDs. The ions/molecules diffusion was effectively hindered by 3 times in the graphene-PCBM EEL in comparison with that of conventional PCBM EEL. The device stability was greatly improved owing to the retardation of ions/molecules diffusion. Meanwhile, the diffusion of electron charge carriers was enhanced to enable a high FF for the device. This work indicates the importance of diffusion engineering for future application of stable and efficient PSCs.

\section{Methods}

Device fabrication. The FTO substrate was first patterned by etching with a $2 \mathrm{M}$ $\mathrm{HCl}$ solution and $\mathrm{Zn}$ powders around a mask formed by strips of adhesive tape. Then, $30 \mathrm{ml}$ of an acetonitrile/ethanol (with 95:5 volume ratio) solution of nickel acetylacetonate (with $15 \mathrm{~mol} \%$ magnesium acetate tetrahydrate and $5 \mathrm{~mol} \%$ lithium acetate) was sprayed by an air nozzle onto the hot FTO glasses at $570{ }^{\circ} \mathrm{C}$. A dense hole transfer layer of $\mathrm{NiMgLiO}$ (about $20 \mathrm{~nm}$ in thickness) was prepared onto the FTO substrate ${ }^{12}$. The prepared MAI and $\mathrm{PbI}_{2}$ for $1.45 \mathrm{M} \mathrm{CH}_{3} \mathrm{NH}_{3} \mathrm{PbI}_{3}$ solution were stirred in DMSO at $70^{\circ} \mathrm{C}$ for $12 \mathrm{~h}$. The resulting solution was coated onto the $\mathrm{NiMgLiO} / \mathrm{FTO}$ substrate by a consecutive spin-coating process at 1,000 and 5,000 r.p.m. for 10 and 20 s, respectively. During the second spin-coating step, the perovskite film was dripped with $1 \mathrm{ml}$ toluene drop-casting. The substrate was thermal treatment on a hotplate at $100^{\circ} \mathrm{C}$ for $10 \mathrm{~min}$. A solution of G-PCBM ( $40 \mathrm{mg} \mathrm{ml}^{-1}$ in chlorobenzene) with $2 \mathrm{wt} \%$ graphene was dissolved at $85^{\circ} \mathrm{C}$ by stirring for $12 \mathrm{~h}$ before use. The synthesis method of functionalized graphene and CQDs were supplied in the Supplementary Information. The G-PCBM with different thickness was deposited on the perovskite/NiMgLiO/FTO substrate by various spin-coating rates for $30 \mathrm{~s}$, with $3,500,2,000,1,500$ and 800 r.p.m. spin-coat rates for 50, 100, 150 and $200 \mathrm{~nm}$ G-PCBM, respectively). In comparison, the PCBM film without graphene addition was also prepared on perovskite/ $\mathrm{NiMgLiO} /$ FTO substrate. After the film was dried at $70{ }^{\circ} \mathrm{C}$ for $10 \mathrm{~min}$, the interfacial layer was prepared from $0.2 \mathrm{mg} \mathrm{ml}^{-1}$ CQDs methanol solution dripping-coating on G-PCBM or PCBM/perovskite/NiMgLiO/FTO substrate at 5,000 r.p.m. for $60 \mathrm{~s}$ The film was dried at $70^{\circ} \mathrm{C}$ for another $10 \mathrm{~min}$. At last, one batch of films was transferred to the evaporator chamber, $100 \mathrm{~nm} \mathrm{Ag}$ or Au/Ag was deposited under high vacuum (lower than $2 \times 10^{-4} \mathrm{~Pa}$ ). The solar cell was sealed by UV glue coating on a cavity glass which covered between the front FTO glass and the active films. All of these processes were done in glove box.

Characterization and measurement. Morphologies of as-obtained products were observed on a field emission scanning electron microscope (SEM, JEOL JSM6500F). Transmission electron microscopy (TEM, JEOL JEM-2100F) images were obtained under an acceleration voltage of $200 \mathrm{kV}$. UV-vis absorption spectra were recorded on a Shimadzu UV 2550 spectrophotometer in the $200-800 \mathrm{~nm}$ wavelength range at room temperature. AFM was performed using Bruker multimode 8 in 'tapping' mode. The crystal structures of perovskite film were characterized by powder X-ray diffraction using a Goniometer Ultima IV (185 nm) diffractometre with $\mathrm{Cu} \mathrm{K}$ radiation, excited at $40 \mathrm{kV}$ and $40 \mathrm{~mA}$. Raman spectra were taken on a DXR Raman Microscope with an excitation length of $532 \mathrm{~nm}$.

Photovoltaic measurement employed a black metal mask with an aperture area of $1.02 \mathrm{~cm}^{2}$ under standard air mass $1.5 \mathrm{G}$ sunlight $\left(100 \mathrm{~mW} \mathrm{~cm}^{-2}\right.$, WXS-155S-10: Wacom Denso Co., Japan) and the simulated light intensity was calibrated with a silicon photodiode. The light soaking stability was tested in a solar cell light resistance test system (Model BIR-50, Bunkoh-Keiki Co., LTD) equipped with a Class AAA solar simulator; the UV light less than $420 \mathrm{~nm}$ was cut off with an optical filter. $J-V$ curves of the PSCs were measured with a digital source metre (Keithley 2400) under simulated solar illumination at $100 \mathrm{~mW} \mathrm{~cm}^{-2}$, AM 1.5G standard and a calibrated Si-reference cell. The $J-V$ curves were measured by reverse (from 1.2 to $-0.2 \mathrm{~V}$ ) or forward (from -0.2 to $1.2 \mathrm{~V}$ ) scan. The step voltage was fixed at $10 \mathrm{mV}$ and the delay time with $50 \mathrm{~ms}$. Monochromatic IPCE spectra were measured with a monochromatic incident light of $1 \times 10^{16}$ photons $\mathrm{cm}^{-2}$ in director current mode (CEP-2000BX, Bunko-Keiki).

The TRPL characterizations were measured on an Edinburg FLS 920 (Edinburg 90 Co. LTD), and the excitation was at the wavelength of $445 \mathrm{~nm}$ with pulse width of 95.3 ps provided by a picosecond pulsed light emitting diode (EPLED-445). Transient photovoltage/photocurrent decay curves were measured on a home-made system with a white light bias generated from an array of diodes $\left(100 \mathrm{~mW} \mathrm{~cm}^{-2}\right)$ and red light pulse diodes $(0.05 \mathrm{~s}$ square pulse width, $100 \mathrm{~ns}$ rise and fall time, $5 \mathrm{~mW} \mathrm{~cm}^{-2}$ ) controlled by a fast solid-state switch were used as the perturbation source. The transient photocurrent was measured using $20 \Omega$ external series resistance to operate the device in short-circuit. Similarly, transient photovoltage was measured using $1 \mathrm{M} \Omega$ external series resistance to operate the device in open-circuit. The voltage dynamics on the resistors were recorded on a digital oscilloscope (Tektronix MDO3032). The perturbation red light source was set to a suitably low level to the white diodes array with light intensity equivalent to $100 \mathrm{~mW} \mathrm{~cm}^{-2}$ of a standard solar simulator.

Data availability. The data that support the findings of this study are available from the corresponding author upon request.

\section{References}

1. Kojima, A., Teshima, K., Shirai, Y. \& Miyasaka, T. Organometal halide perovskites as visible-light sensitizers for photovoltaic cells. J. Am. Chem. Soc. 131, 6050-6051 (2009)

2. Dong, Q. et al. Electron-hole diffusion lengths $>175 \mu \mathrm{m}$ in solution-grown $\mathrm{CH}_{3} \mathrm{NH}_{3} \mathrm{PbI}_{3}$ single crystals. Science 347, 967-970 (2015).

3. Stranks, S. D. et al. Electron-hole diffusion lengths exceeding 1 micrometer in an organometal trihalide perovskite absorber. Science 342, 341-344 (2013).

4. Xing, G. et al. Long-range balanced electron-and hole-transport lengths in organic-inorganic $\mathrm{CH}_{3} \mathrm{NH}_{3} \mathrm{PbI}_{3}$. Science 342, 344-347 (2013).

5. Kim, H.-S. et al. Lead iodide perovskite sensitized all-solid-state submicron thin film mesoscopic solar cell with efficiency exceeding 9\%. Sci. Rep. 2, 591 (2012). 
6. Lee, M. M., Teuscher, J., Miyasaka, T., Murakami, T. N. \& Snaith, H. J. Efficient hybrid solar cells based on meso-superstructured organometal halide perovskites. Science 338, 643-647 (2012).

7. Liu, M., Johnston, M. B. \& Snaith, H. J. Efficient planar heterojunction perovskite solar cells by vapour deposition. Nature 501, 395-398 (2013).

8. Zhou, H. et al. Interface engineering of highly efficient perovskite solar cells. Science 345, 542-546 (2014).

9. Mei, A. et al. A hole-conductor-free, fully printable mesoscopic perovskite solar cell with high stability. Science 345, 295-298 (2014).

10. Yang, W. S. et al. High-performance photovoltaic perovskite layers fabricated through intramolecular exchange. Science 348, 1234-1237 (2015).

11. Jeon, N. J. et al. Compositional engineering of perovskite materials for high-performance solar cells. Nature 517, 476-480 (2015).

12. Chen, W. et al. Efficient and stable large-area perovskite solar cells with inorganic charge extraction layers. Science 350, 944-948 (2015).

13. Li, X. et al. A vacuum flash-assisted solution process for high-efficiency large-area perovskite solar cells. Science 353, 58-62 (2016).

14. Green, M. A., Emery, K., Hishikawa, Y., Warta, W. \& Dunlop, E. D. Solar cell efficiency tables (version 48). Prog. Photovolt. 24, 905-913 (2016).

15. McMeekin, D. P. et al. A mixed-cation lead mixed-halide perovskite absorber for tandem solar cells. Science 351, 151-155 (2016).

16. Kato, Y. et al. Silver iodide formation in methyl ammonium lead iodide perovskite solar cells with silver top electrodes. Adv. Mater. Interfaces 2, 1500195 (2015).

17. Li, X. et al. Improved performance and stability of perovskite solar cells by crystal crosslinking with alkylphosphonic acid $\omega$-ammonium chlorides. Nat. Chem. 7, 703-711 (2015).

18. Leijtens, T. et al. Stability of metal halide perovskite solar cells. Adv. Energy Mater. 5, 23 (2015)

19. You, J. et al. Improved air stability of perovskite solar cells via solutionprocessed metal oxide transport layers. Nat. Nanotechnol. 11, 75-81 (2016).

20. Liu, J. et al. A dopant-free hole-transporting material for efficient and stable perovskite solar cells. Energy Environ. Sci. 7, 2963-2967 (2014).

21. Leijtens, T. et al. Overcoming ultraviolet light instability of sensitized $\mathrm{TiO}_{2}$ with meso-superstructured organometal tri-halide perovskite solar cells. Nat. Commun. 4, 2885 (2013).

22. Conings, B. et al. Intrinsic thermal instability of methylammonium lead trihalide perovskite. Adv. Energy Mater. 5, 8 (2015).

23. Dualeh, A., Gao, P., Seok, S. I., Nazeeruddin, M. K. \& Grätzel, M. Thermal behavior of methylammonium lead-trihalide perovskite photovoltaic light harvesters. Chem. Mat. 26, 6160-6164 (2014).

24. Abate, A. et al. Supramolecular halogen bond passivation of organic-inorganic halide perovskite solar cells. Nano. Lett. 14, 3247-3254 (2014).

25. Zheng, L. et al. A hydrophobic hole transporting oligothiophene for planar perovskite solar cells with improved stability. Chem. Commun. 50, 11196-11199 (2014)

26. Bi, C. et al. Non-wetting surface-driven high-aspect-ratio crystalline grain growth for efficient hybrid perovskite solar cells. Nat. Commun. 6, 7 (2015).

27. Eames, C. et al. Ionic transport in hybrid lead iodide perovskite solar cells Nat. Commun. 6, 8 (2015).

28. Shao, Y., Xiao, Z., Bi, C., Yuan, Y. \& Huang, J. Origin and elimination of photocurrent hysteresis by fullerene passivation in $\mathrm{CH}_{3} \mathrm{NH}_{3} \mathrm{PbI}_{3}$ planar heterojunction solar cells. Nat. Commun. 5, 7 (2014).

29. Haruyama, J., Sodeyama, K., Han, L. Y. \& Tateyama, Y. First-principles study of ion diffusion in perovskite solar cell sensitizers. J. Am. Chem. Soc. 137, 10048-10051 (2015)

30. Delugas, P., Caddeo, C., Filippetti, A. \& Mattoni, A. Thermally activated point defect diffusion in methylammonium lead trihalide: anisotropic and ultrahigh mobility of iodine. J. Phys. Chem. Lett. 7, 2356-2361 (2016).

31. Zhang, G. Y. et al. Extensive penetration of evaporated electrode metals into fullerene films: intercalated metal nanostructures and influence on device architecture. ACS Appl. Mater. Interfaces 7, 25247-25258 (2015).

32. Green, M. A., Emery, K., Hishikawa, Y., Warta, W. \& Dunlop, E. D. Solar cell efficiency tables (version 47). Prog. Photovolt. 24, 3-11 (2016).

33. Chen, W. et al. Hybrid interfacial layer leads to solid performance improvement of inverted perovskite solar cells. Energy Environ. Sci. 8, 629-640 (2015).

34. Jun, G. H. et al. Enhanced conduction and charge-selectivity by N-doped graphene flakes in the active layer of bulk-heterojunction organic solar cells. Energy Environ. Sci. 6, 3000-3006 (2013).

35. Qu, S. et al. Noncovalent functionalization of graphene attaching $[6,6]$-phenyl-C61-butyric acid methyl ester (PCBM) and application as electron extraction layer of polymer solar cells. ACS Nano 7, 4070-4081 (2013).

36. Sun, K., Chang, J., Isikgor, F. H., Li, P. \& Ouyang, J. Efficiency enhancement of planar perovskite solar cells by adding zwitterion/LiF double interlayers for electron collection. Nanoscale 7, 896-900 (2015).

\section{Acknowledgements}

This work was supported by the National Natural Science Foundation of China (grant no. 11574199, 11674219 and 51402190), the Programme for Professor of Special Appointment (Eastern Scholar) at Shanghai Institutions of Higher Learning, the New Energy and Industrial Technology Development Organization (NEDO), the Pudong New Area Science and Technology Development Innovation Fund (grant no. PKJ2014-Z02), the third 211 Project (WS3116205009) of Shanghai Jiao Tong University, and Shanghai Natural Science Foundation (no. 13ZR1456600). The authors thank T. Shimizu and T. Ishikawa for technical support.

\section{Author contributions}

E.B., C.H. and F.X. contributed to all the experimental work. X.Y., E.B. and F.X. analysed the data. Y.W. contributed to the deposition of electron extraction layer. W.C. contributed to the deposition of hole extraction layer. H.C. and A.I. contributed to the characterization of device performance. Y.S. contributed to the synthesis of carbon quantum dots. X.Y. and L.H. designed and directed the study. X.Y., M.G. and L.H. wrote the manuscript. All authors reviewed the paper.

\section{Additional information}

Supplementary Information accompanies this paper at http://www.nature.com/ naturecommunications

Competing interests: The authors declare no competing financial interests.

Reprints and permission information is available online at http://npg.nature.com/ reprintsandpermissions/

How to cite this article: Bi, E. et al. Diffusion engineering of ions and charge carriers for stable efficient perovskite solar cells. Nat. Commun. 8, 15330 doi: 10.1038/ncomms15330 (2017)

Publisher's note: Springer Nature remains neutral with regard to jurisdictional claims in published maps and institutional affiliations.

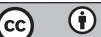

This work is licensed under a Creative Commons Attribution 4.0 International License. The images or other third party material in this article are included in the article's Creative Commons license, unless indicated otherwise in the credit line; if the material is not included under the Creative Commons license, users will need to obtain permission from the license holder to reproduce the material. To view a copy of this license, visit http://creativecommons.org/licenses/by/4.0/

(C) The Author(s) 2017 